\title{
Computation of Errors and Their Analysis On Physics Experiments
}

\author{
Khadka Bahadur Chhetri \\ Department of Physics, Prithvi Narayan Campus, Tribhuvan University, Nepal \\ Correspondence:kckhadak@yahoo.com
}

\begin{abstract}
The process of evaluating uncertainty associated with a measurement result is often called uncertainty analysis or error analysis. Without proper error analysis, no valid scientific conclusions can be drawn. The uncertainty of a single measurement is limited by the precision and accuracy of the measurement. The errors are communicated in different mathematical operations. Every deviation in result of experiment from the expected one has important significance. Even on undergraduate level of our domestic universities, error analysis and interpretation of the result should be done instead of writing just the points of precautions. This approach will make the students somewhat familiar about the error analysis on advanced level. Otherwise, the hesitation and difficulty about error analysis which stuns M.Sc. level beginner student will persist and the necessity of standardization of our lab reports will remain untouched for next several years also.
\end{abstract}

Keywords: computation errors, degree of uncertainty, precise measurment.

\section{Introduction}

All measurements have some degree of uncertainty that may come from a variety of sources. The process of evaluating this uncertainty associated withameasurementresult is oftencalleduncertainty analysis or error analysis. Error analysis may seem tedious; however, without proper error analysis, no valid scientific conclusions can be drawn. In fact, bad things can happen if error analysis is ignored. The failure to specify the error for a given measurement can have serious consequences in science and in real life. Since there is no way to avoid error analysis, it is best to learn how to do it right. Error in a scientific measurement usually does not mean a mistake or blunder. Instead, the terms "error" and "uncertainty" both refer to unavoidable imprecision in measurements.

The uncertainty of a single measurement is limited by the precision and accuracy of the measuring instrument, along with any other factors that might affect the ability of the experimenter to make the measurement. In order to determine the accuracy of a particular measurement, we have to know the true value. Sometimes we have a "textbook" measured value which is known precisely, and we assume that this is our "ideal" value, and use it to estimate the accuracy of our result. Other times we know a theoretical value which is calculated from basic principles, and this also may be taken as an "ideal" value. The main purpose of error analysis is to check whether the result of experiment agrees with a theoretical prediction or results from other experiments or not. Generally speaking, a measured result agrees with a theoretical prediction if the prediction lies within the range of experimental uncertainty.

\section{Definition of Errors}

Difference between measured value and true value of a quantity represents error of measurement.

\section{Accuracy and Errors in a measurement:}

The accuracy of a measurement is limited by the least count of the measuring instrument and by minimizing Errors.

\section{Types of Errors}

1. Constant Errors: The errors due to constant causes are called constant errors. Ex: due to faulty calibration of a measuring instrument. Such faulty instruments should be discarded as possible as.

2. Systematic Errors: The error which is governed by some systematic rule is called 
systematic error. Ex: zero error on screw gauge, index error of optical bench... etc. Such errors can be reduced by associated systematic rules.

3. Random Errors: The errors occurring due to unknown reasons are called random error. Ex: Error due change in condition of experiment, incorrect judgment of an observer in making measurement... etc. Such errors can be minimized by repeating experiment many times and taking arithmetic mean.

\section{Common sources of error in physics laboratory experiments}

Incomplete definition(may be systematic or random) : carefully consider and specify the conditions that could affect the measurement.

Failure to account for a factor (usually systematic): Care/account about all possible implicit and explicit factors should be done before beginning the experiment so that arrangements can be made to account for the confounding factors before taking data.

Environmental factors(systematic or random) :Be aware of errors introduced by your immediate working environment.

Instrument resolution(random) - All instruments have finite precision that limits the ability to resolve small measurement differences.

Failure to calibrate or check zero of instrument(systematic) - Whenever possible, the calibration of an instrument should be checked before taking data.

Physical variations(random) - It is always wise to obtain multiple measurements over the entire range being investigated.

Parallax (systematic or random) - If the observer's eye is not squarely aligned with the pointer and scale, the reading may be too high or low.

Instrument drift (systematic) - Most electronic instruments have readings that drift over time. Occasionally this source of error can be significant and should be considered.

Lag time and hysteresis (systematic) - Some measuring devices require time to reach equilibrium, and taking a measurement before the instrument is stable will result in a measurement that is generally too low. The most common example is taking temperature readings with a thermometer that has not reached thermal equilibrium with its environment. A similar effect is hysteresis where the instrument readings lag behind and appear to have a "memory" effect as data are taken sequentially moving up or down through a range of values. Hysteresis is most commonly associated with materials that become magnetized when a changing magnetic field is applied.

Personal errors: Gross personal errors, sometimes called mistakes or blunders, should be avoided and corrected if discovered.

\section{Absolute and Relative errors}

Let $x_{1}, x_{2}, x_{3} x_{n}$ be the $n$ measurements of a physical quantity. Then A. M. of measurement $\bar{x} \bar{x}=\frac{x_{2}+x_{2}+x_{2}+\cdots+x_{n} x_{2}+x_{2}+x_{2}+\cdots+x_{n}}{n}=\frac{\sum x_{1} \sum x_{1}}{n n} \quad$ TThis is called true value]

Absolute error: $\Delta \mathrm{x}=$ measured value - true value

$$
=x_{i}-\bar{x} x_{i}-\bar{x}
$$

Final absolute error:

$\Delta \bar{x} \bar{x}=\frac{\left|\Delta x_{2}\right|+\left|\Delta x_{2}\right|+\left|\Delta x_{2}\right||\ldots \ldots+\ldots+| \Delta x_{n}|| \Delta x_{2}|+| \Delta x_{2}|+| \Delta x_{2}|\ldots \ldots \ldots+| \Delta x_{n} \mid}{n}=$ $\frac{\sum\left|\Delta x_{1}\right| \sum\left|\Delta x_{1}\right|}{n} n$

Final result $=\bar{x} \pm \bar{x} \pm \Delta \bar{x} \bar{x}$

Relative error $=\frac{\Delta x \Delta x}{\bar{x} x}$

$\%$ error $=\frac{\Delta x}{\bar{x}} \times 100 \% \frac{\Delta x}{\bar{x}} \times 100 \%$

\section{Combination(propagation) of Errors:}

The errors are communicated in different

mathematical operations.

\section{i. Errors in sum or Difference:}

a. In Sum:

Let $X=(a+b)$ be the quantity. If $\Delta a \Delta a$ is error in 
measurement of ' $a$ ', $\Delta b \Delta b$ be that in ' $b$ ', then

Permissible error in $\mathrm{X}=\Delta a+\Delta b \Delta a+\Delta b$

Relative error $=\frac{\Delta x}{x}=\frac{\Delta a+\Delta e \Delta x}{a+b x}=\frac{\Delta a+\Delta e}{a+b}$

$\%$ error $=\frac{\Delta x}{x} \times 100 \%=\frac{\Delta a+\Delta b}{a+b} \times 100 \%$

$\frac{\Delta x}{x} \times 100 \%=\frac{\Delta a+\Delta b}{a+b} \times 100 \%$

b. In Difference:

Let $\mathrm{X}=(\mathrm{a}-\mathrm{b})$ be the quantity. If $\Delta a \Delta a$ is error in measurement of ' $a$ ', $\Delta b \Delta b$ be that in 'b', then

Permissible error in $\mathrm{X}=\Delta a+\Delta b \Delta a+\Delta b[$ Since,

error is always additive]

Relative error $=\frac{\Delta x}{x}=\frac{\Delta a+\Delta e \Delta x}{a-b x}=\frac{\Delta a+\Delta e}{a-b}$

$\%$ error $=\frac{\Delta X}{x} \times 100 \%=\frac{\Delta a+\Delta D}{a-b} \times 100 \%$

$\frac{\Delta x}{x} \times 100 \%=\frac{\Delta a+\Delta b}{a-b} \times 100 \%$

ii. Errors in Product and Quotient:

a. In Product:

Let $X=\frac{a a}{b \dot{b}}$ be the quantity. If $\Delta a \Delta a$ is error in measurement of ' $a$ ', $\Delta b \Delta b$ be that in 'b', then

Taking $\log : \log \mathrm{X}=\log \mathrm{a}--\log \mathrm{b}$

Differentiating we get; $\frac{\Delta X}{x}=\frac{\Delta a}{a}-\frac{\Delta \Delta \Delta x}{b x}=\frac{\Delta a}{a}-\frac{\Delta a}{b}$

Permissible error in $\mathrm{X}=\Delta X \Delta X=\mathrm{X}\left(\frac{\Delta a}{a}+\frac{\Delta o \Delta a}{b a}+\frac{\Delta a}{b}\right)=$ $\mathrm{ab}\left(\frac{\Delta a}{a}+\frac{\Delta \Delta \Delta a}{b a}+\frac{\Delta a}{b}\right)$ [Since, error is additive]

Relative error $=\frac{\Delta X \Delta x}{X X}=\frac{\Delta a}{a}+\frac{\Delta \theta \Delta a}{b a}+\frac{\Delta o}{b}$

$\%$ error $=\frac{\Delta X}{X} \times 100 \%=\left(\frac{\Delta a}{a}+\frac{\Delta D}{b}\right) \times 100 \%$

$\frac{\Delta X}{X} \times 100 \%=\left(\frac{\Delta a}{a}+\frac{\Delta D}{b}\right) \times 100 \%$

b. In Quotient:

Let $X=a b$ be the quantity. If $\Delta a \Delta a$ is error in measurement of 'a', $\Delta b \Delta b$ be that in 'b', then

Taking $\log : \log \mathrm{X}=\log \mathrm{a}+\log \mathrm{b}$

Differentiating we get; $\frac{\Delta x}{x}=\frac{\Delta a}{a}+\frac{\Delta D}{b} \frac{\Delta x}{x}=\frac{\Delta a}{a}+\frac{\Delta b}{b}$

Permissible error in $\mathrm{X}=\Delta X \Delta X=\mathrm{X}\left(\frac{\Delta a}{a}+\frac{\Delta \theta \Delta a}{b a}+\frac{\Delta a}{b}\right)$
$=\mathrm{ab}\left(\frac{\Delta a}{a}+\frac{\Delta \Delta \Delta a}{b a}+\frac{\Delta a}{b}\right)$

Relative error $=\frac{\Delta x \Delta x}{x x}=\frac{\Delta a}{a}+\frac{\Delta \theta \Delta a}{b a}+\frac{\Delta a}{b}$

$\%$ error $=\frac{\Delta X}{x} \times 100 \%=\left(\frac{\Delta a}{a}+\frac{\Delta D}{b}\right) \times 100 \%$

$\frac{\Delta X}{X} \times 100 \%=\left(\frac{\Delta a}{a}+\frac{\Delta D}{b}\right) \times 100 \%$

\section{iii. Errors due to raised power:}

Let $\mathrm{X}=K \frac{\mathrm{a}^{\mathrm{n}} \mathrm{b}^{\mathrm{m}}}{c^{\mathrm{y}}} K \frac{\mathrm{a}^{\mathrm{n}} \mathrm{b}^{\mathrm{m}}}{c^{\mathrm{y}}}$ be the quantity, where $\mathrm{K}$ is a constant. If $\Delta a \Delta a$ is error in measurement of ' $\mathrm{a}$ ' $\Delta b \Delta b$ be that in 'b' and $\Delta c \Delta c$ that in 'c' then

Taking log:

$\log \mathrm{X}=\log \mathrm{K}+\mathrm{n} \log \mathrm{a}+\operatorname{mlog} \mathrm{b}-\operatorname{plog} \mathrm{c}$

Differentiating we get;

$\left.\frac{\Delta X}{X}=n \frac{\Delta a}{a}+m \frac{\Delta b}{b}-p \frac{\Delta c}{c}\right)$

[Since, no error is involved in a constant]

Permissible error in $\mathrm{X}=\Delta X \Delta X$

$=\mathrm{X}\left(\mathrm{n} \frac{\Delta a}{a}+m \frac{\Delta b}{b}+p \frac{\Delta c \Delta a}{c a}+m \frac{\Delta b}{b}+p \frac{\Delta c}{c}\right)$

$=\mathrm{ab}\left(\mathrm{n} \frac{\Delta a}{a}+m \frac{\Delta b}{b}+p \frac{\Delta c \Delta a}{c a}+m \frac{\Delta b}{b}+p \frac{\Delta a}{c}\right)$

Relative error $=\frac{\Delta X \Delta X}{X X}=\left(n \frac{\Delta a}{a}+m \frac{\Delta b}{b}+p \frac{\Delta c}{c}\right)$

$\left(n \frac{\Delta a}{a}+m \frac{\Delta b}{b}+p \frac{\Delta c}{c}\right)$

$\%$ error $=\frac{\Delta X}{v} \times 100 \% \frac{\Delta X}{v} \times 100 \%$

$=\left(n \frac{\Delta a}{a}+m \frac{\Delta b}{b}+p \frac{\Delta c}{c}\right) \times 100 \%$

\section{Significant Figures}

In the measured value of a physical quantity, the digits about the correctness of which we are sure plus the last digit which is doubtful are called significant figures.

\section{Rules for counting significant figures}

i. All the non-zero digits are significant. Ex: 98576 has five significant digits.

ii. All zeros occurring in between the two nonzero digits are significant. Ex: 90008 has five significant digits.

iii. In a number without decimal, zeros on the 
right of non-zero digit are not significant. Ex: 7890 has only three significant digits.

iv. In a number with decimal, zeros on the right of the last non zero digit are significant. Ex: 9.78000 has six significant digits.

v. In a value less than one, zeros occurring between the decimal point and non-zero digit on the right are not significant. Ex: 0.00987 has only three significant digits.

On Brief: Front zeros are always not significant, middle zeros are always significant and last zeros are significant only if there is decimal point.

\section{Remarks}

i. The change in units of measurement of a quantity does not affect the number of significant figures.

ii. When one value is expressed in an exponential form, the exponential term does not affect the number of significant figures. Ex: $98.7=$ $0.0987 \times 10^{3}=98700 \times 10^{-3}$ have only three significant figures. [The powers of 10 are not counted as significant figures.]

iii. The error in measurement is equal to least count (L.C.) of measuring instrument.

iv. If least count of measuring instrument is not given, then error is $\pm 1 \pm 1$ in the last digit of measurement.

v. Greater the number of significant figures in a measurement, smaller is the percentage error.

vi. Never round off intermediate results when performing a chain of calculations. The associated round-off errors can quickly propagate and cause the final result to be unnecessarily inaccurate. So, keep as many digits as is practical to avoid rounding off errors. Take two more significant figures than required and at the end, round off the final result to a reasonable number of significant figures.

Mathematical Operations and Significant Figures

a. Addition and subtraction:

The result contains the minimum numbers of decimal places in any of the quantities involved. Ex: $34.321+5.4=39.721 \approx \approx 39.7$ [Rounding off on first place of decimal, since, 5.4 has only one digit after decimal]

\section{b. Multiplication and Division:}

The result contains the minimum number of significant figures in any of the quantities involved.

Ex: $\frac{194.56}{3.3}=58.957 \approx 5 \frac{194.56}{3.3}=58.957 \approx 59$ [Rounding off on nil place of decimal, since, 3.3 has only two significant figures]

\section{Rounding Off:}

The process of cutting off superfluous digit and retaining as many desired is called rounding off.

In rounding off process if the digit next to one to be more than 5 , the digit next to one to be rounded off is increased by 1 ; if it is less than 5 , digit to be rounded is left is left unchanged. But if the next digit is exactly 5 , then the digit to be rounded is increased by 1 if it is odd and keep unchanged if it is even. This can be remembered by the concept that we physicists always prefer even parity or symmetry. Ex: Let's round off the following numbers on second decimal position.

$$
\begin{aligned}
& 5.478 \approx \approx 5.48 \\
& 5.473 \approx \approx 5.47 \\
& 5.475 \approx \approx 5.48 \\
& 5.465 \approx \approx 5.46
\end{aligned}
$$

[Note: While expressing final result of a measurement/experiment, the measurement must be expressed upto proper significant figures, depending upon the least count of your measuring instrument and order of expected result.]

\section{Examples of Error Analysis in Experiments:}

\section{Ex 1.: Fresnel's Bi-prism}

Object of the Experiment: To determine the wavelength of the light wave emitted by the given light source.

Formula Used: $\lambda=\frac{\beta d \beta d}{D D}$; where, $\beta=$ fringe width, $\mathrm{d}=$ slit separation and $\mathrm{D}=$ distance between slit $\&$ source. 
Calculation:

$$
\begin{aligned}
\lambda_{i} \lambda_{i}=\left[\frac{\beta}{D} \bar{d} \frac{\beta}{D} \bar{d}\right]_{i} \\
\quad \therefore \bar{\lambda}: \bar{\lambda}=\frac{\sum \lambda_{i}}{n} ; \frac{\sum \lambda_{i}}{n} ; \text { for different D }
\end{aligned}
$$

Error Analysis

$\Delta \lambda \Delta \lambda=\lambda \sqrt{\left[\left(\frac{\Delta \beta}{\beta}\right)^{2}+\left(\frac{\Delta d}{d}\right)^{2}+\left(\frac{\Delta D}{D}\right)^{2}\right]}$

$\lambda \sqrt{\left[\left(\frac{\Delta \beta}{\beta}\right)^{2}+\left(\frac{\Delta d}{d}\right)^{2}+\left(\frac{\Delta D}{D}\right)^{2}\right]}$

\{Refer: Errors in Product andQuotient\}

$\Rightarrow(\Delta \lambda)^{2} \Rightarrow(\Delta \lambda)^{2}=\lambda^{2}\left[\left(\frac{\Delta \beta}{\beta}\right)^{2}+\left(\frac{\Delta d}{d}\right)^{2}+\left(\frac{\Delta D}{D}\right)^{2}\right]$

$\lambda^{2}\left[\left(\frac{\Delta \beta}{\beta}\right)^{2}+\left(\frac{\Delta d}{d}\right)^{2}+\left(\frac{\Delta D}{D}\right)^{2}\right]$

Since, $\lambda=\lambda(\beta, d, D) \lambda=\lambda(\beta, d, D)$ and correlation coeff. for independent variables is zero i.e. product of

average mean deviations of independent variables is zero $\}$

Or, $\Delta \lambda \Delta \lambda=\lambda \sqrt{\left[\left(\frac{\Delta \beta}{\beta}\right)^{2}+\left(\frac{\Delta d}{d}\right)^{2}+\left(\frac{\Delta D}{D}\right)^{2}\right]}$

$\lambda \sqrt{\left[\left(\frac{\Delta \beta}{\beta}\right)^{2}+\left(\frac{\Delta d}{d}\right)^{2}+\left(\frac{\Delta D}{D}\right)^{2}\right]}$

Where,

a. $\beta=\frac{0-a}{n} \beta=\frac{0-a}{n} \quad$ i.e. $\beta=\beta(b, a, n)$ $\beta=\beta(b, a, n)$

Or, $\Delta \beta \Delta \beta=\beta \sqrt{\left[\left(\frac{\Delta b}{b}\right)^{2}+\left(\frac{\Delta a}{a}\right)^{2}+\left(\frac{\Delta n}{n}\right)^{2}\right]}$

$\beta \sqrt{\left[\left(\frac{\Delta b}{b}\right)^{2}+\left(\frac{\Delta a}{a}\right)^{2}+\left(\frac{\Delta n}{n}\right)^{2}\right]}$

[On calculation, take $\frac{\Delta n}{n}=0 \frac{\Delta n}{n}=0$ because for distinct Eigen states $\Delta n=0 \Delta n=0]$

[Note: Don't hesitate about the relation since cross terms are absent.]

b. $\quad d=\sqrt{d_{1} d_{2}} d=\sqrt{d_{1} d_{2}}=\left[d_{1} d_{2}\right]^{\frac{2}{2}}\left[d_{1} d_{2}\right]^{\frac{2}{2}}$
Taking logarithm and diff. we get,

$(\Delta d)^{2}(\Delta d)^{2}=\frac{1}{4} d^{2}\left[\left(\frac{\Delta d_{2}}{d}\right)^{2}+\left(\frac{\Delta d_{2}}{d}\right)^{2}\right] \frac{1}{4} d^{2}\left[\left(\frac{\Delta d_{2}}{d}\right)^{2}+\left(\frac{\Delta d_{2}}{d}\right)^{2}\right]$

Methods to choose values of $\underline{\Delta \beta \Delta} \underline{\beta}, \underline{\underline{\Delta d} \Delta d}$ for the calculation of $\underline{\Delta \lambda \Delta \lambda}$ :

We take more than three observations for any observable like $\beta, d \beta, d \ldots$ etc. Therefore, we adopt different methods to choose values of $\Delta \beta \Delta \beta, \Delta d \Delta d$ for the calculation of $\Delta \lambda \Delta \lambda$.

A. Method first: (Method of maxm. probable error)

$\Delta \beta=\operatorname{maxm}\left(\Delta \beta_{i}\right)$

$\Delta d=\operatorname{maxm} \cdot\left(\Delta d_{i}\right)$

$\Delta D=\Delta D=$ Least Count.

[Since, D is individually treated]

B. Method second:(Method of Std. deviation)

$$
\begin{gathered}
\Delta \beta=\frac{\sigma_{\beta}}{\sqrt{n}} \text { Where, } \sigma_{x}=\frac{1}{n} \sum f_{i}\left(x_{i}-\bar{x}\right)^{2} \\
\Delta d=\frac{\sigma_{d}}{\sqrt{n}}
\end{gathered}
$$

$\Delta D=$ Least Count

[Since, D is individually treated]

C. Method third: (Method of weighted errors)

This method helps to minimize error due to nonuniform scaling on measurements. So, this method is best to use in case of non-uniform scaling.

Ex: Both $\Delta d$ and $\Delta D=0.01 \mathrm{~cm}$

$\Delta d$ and $\Delta D=0.01 \mathrm{~cm}$

where $d \approx 1 \mathrm{~cm}$ whereas $D \approx 100 \mathrm{~cm}$.

Let, $\quad \Delta \beta_{1} \Delta \beta_{1}=\mathrm{r}_{1}$

$\Delta \beta_{2} \Delta \beta_{2}=r_{2}$

$\Delta \beta_{3} \Delta \beta_{3}=r_{3}$ 
$\Delta \beta_{1} \Delta \beta_{1}=\mathrm{r}_{4}$ and

$\Delta \beta_{5} \Delta \beta_{5}=\mathrm{r}_{5}$,

Then,$\frac{1}{r_{1}{ }^{2} w_{1}}=\frac{1}{r_{2}{ }^{2} w_{2}}=\frac{1}{r_{3}{ }^{2} w_{3}}=\frac{1}{r_{4}{ }^{2} w_{4}}=\frac{1}{r_{5}{ }^{2} w_{5}}=$ const.

$\frac{1}{r_{1}^{2} w_{1}}=\frac{1}{r_{2}^{2} w_{2}}=\frac{1}{r_{3}^{2} w_{3}}=\frac{1}{r_{4}{ }^{2} w_{4}}=\frac{1}{r_{5}^{2} w_{5}}=$ const.

Where, $w_{i} w_{i}=$ considered weight of the $i^{\text {th }}$ prob. error.

Suppose, $w_{1}=1 w_{1}=1 \quad$ (You take unit value for anyone).

And calculate $w_{2}, w_{3}, w_{4}$ and $w_{5} w_{2}, w_{3}, w_{4}$ and $w_{5}$ using above parametric equation.

[Note: for reliable

$\left\{0.8 \leq w_{i} \leq 1.2\right\}$ ie.with $\pm 20 \%$

$\left\{0.8 \leq w_{i} \leq 1.2\right\}$ ie.with $\pm 20 \%$; with any one $w_{i}=1$ $w_{i}=1$ It is better to discard unreliable data as possible as, since they assist on increment of random error.]

Then, $\bar{\beta}_{1} \bar{\beta}_{1}=\frac{\sum w_{1} \beta \sum w_{t} \beta}{\sum w_{1}} \frac{\sum w_{1}}{w_{1}}$

- $1 \beta_{w}=\frac{\left[\max \cdot\left(\Delta^{1} \beta_{i}\right)\right]}{\sqrt{\sum w_{i}}}$ le error on $\beta \beta$ is;

$=\bar{\beta}_{w}+\frac{\left[\max \cdot\left(\Delta \beta_{i}\right)\right] \mathrm{st}}{\sqrt{\sum w_{i}}}$ probable value of $\beta \beta$

Similarly, do the same processes to calculate most prob. value of $d$.

But, most prob. value of $\mathrm{D}=\mathrm{D}+\Delta \Delta \mathrm{D}$

[Since D is individually treated.]

Then, $\Delta \lambda \Delta \lambda=\bar{\lambda} \sqrt{\left[\left(\frac{\Delta \beta_{w}}{\beta_{w}}\right)^{2}+\left(\frac{\Delta d_{w}}{d_{w}}\right)^{2}+\left(\frac{\Delta D}{D}\right)^{2}\right]}$

$$
\bar{\lambda} \sqrt{\left[\left(\frac{\Delta \beta_{w}}{\beta_{w}}\right)^{2}+\left(\frac{\Delta d_{w}}{d_{w}}\right)^{2}+\left(\frac{\Delta D}{D}\right)^{2}\right]}
$$

D. Method fourth:(Method of least square fitting): (For the equations which can be transformed in the $y=m x+c$ form)

\section{Ex 2.: Radioactive sample}

Object of the Experiment: To determine the half life of given radioactive sample.

Formula used:

$$
\mathrm{N}=\mathrm{N}_{0} e^{-\lambda t_{e}}-\lambda t
$$

Where, $\mathrm{N}_{0}=$ numbers of atom present at time $\mathrm{t}=0$.

\section{Calculation}

Taking logarithm on both sides, we get, $\ln \mathrm{N}=$ $\ln \mathrm{N}_{0}-\lambda t-\lambda t$

Now, this equation is in the form of straight line.

So, comparing with $\mathrm{y}=\mathrm{mx}+\mathrm{c}$, we get,

$\mathrm{y}=\ln \mathrm{N}, \mathrm{x}=\mathrm{t}, \mathrm{m}=-\lambda-\lambda$ and $\mathrm{c}=\ln \mathrm{N}_{0}$

Now, taking time of counts on $\mathrm{x}$ - axis, logarithm

of no. of decaying atoms per second on y-axis, we can determine $\lambda \lambda$ and $\mathrm{N}_{0}$ by finding slope and $y$-intercept of st. line on plotting graph of $\ln N$ versus t.

At the same time we also calculate $\mathrm{m}$ and $\mathrm{c}$ by the method of least square fitting i.e from the normal equations:

$\sum y=m \sum x+n c \sum y=m \sum x+n c$ and

$\sum x y=m \sum x^{2}+c \sum x \sum x y=m \sum x^{2}+c \sum x$.

Solving these two equations we can obtain the values of $\mathrm{m}$ and $\mathrm{C}$.

Hence, using, $T_{\frac{2}{2}}=\frac{\ln 2}{\lambda} T_{\frac{2}{2}}=\frac{\ln 2}{\lambda}$, we can determine the half life time of the given radioactive substance.

The probable error on slope $(\mathrm{m})$ is:

$$
\begin{aligned}
& \Delta m=0.6745 \sqrt{\frac{n \sum\left(e_{y}\right)^{2}}{(n-2)\left[n \sum x^{2}-\left(\sum x\right)^{2}\right]}} \\
& \Delta C=0.6745 \sqrt{\frac{\sum x^{2} \sum\left(e_{y}\right)^{2}}{(n-2)\left[n \sum x^{2}-\left(\sum x\right)^{2}\right]}} \mathrm{t}(\mathrm{c}) \text { is: }
\end{aligned}
$$

(Where, $e_{y=} y_{\text {obs }}-y_{\text {calc }}{ }^{e} y=y_{o b s}-y_{\text {calc }}$ ) 
[Note: Prob. Error $=0.6745 \times$ Std. Error]

Since, we have $m=-\lambda \therefore \Delta m=\Delta \lambda-\lambda \therefore \Delta m=\Delta \lambda$ on magnitude.

Again, since $\mathrm{c}=\ln \mathrm{N}_{0}$

$\therefore \Delta C=\frac{\Delta N_{0}}{N_{0}} \quad \therefore \Delta C=\frac{\Delta N_{0}}{N_{0}} \quad$ i.e $\quad \therefore \Delta N_{0}=\Delta C \cdot N_{0}$

$\therefore \Delta N_{0}=\Delta C \cdot N_{0}$

E. Method Fifth:(Method of $\chi^{2}-$ test

Method Fifth:(Method of $\chi^{2}-$ test)

$\chi_{\text {calc. }}^{2}=\sum_{i} \frac{\left(e_{i}-o_{i}\right)^{2}}{o_{i}}$

Degrees of freedom $(\mathrm{f})=\mathrm{n}-1$

(Where, $\mathrm{n}=$ total no. of observations)

$$
\chi_{\text {tab. }}^{2}=\text { ? }
$$

[Observe table of $\chi^{2}-$ testof $\chi^{2}-$ test for $\mathrm{f}$ degrees of freedoms to find if your data are correct at $99 \%$ confidence level i.e.at $1 \%$ level of significance or $95 \%$ confidence level i.e.at 5\% level of significance. Your data should be precise within these levels of confidence.]

If $\chi_{\text {calc. }}^{2}<\chi_{\text {tab }}^{2} \chi_{\text {calc. }}^{2}<\chi_{\text {tab. }}^{2}$ then we say that the observed values are close to expected values with the ...\% confidence level.

\section{Discussion of the Result of the Experiment}

After computation of the value of the physical quantity you must interpret your result. This section is included as Discussion section on filing the experiment you did in your lab. There, you should state how precise and how accurate your result is. You should discuss the sources responsible for error on your result. Not only this, you must discuss about the significance of your result. Every deviation in result from the expected one has important significance.

For example: On sugar solution experiment for determination of refractive index at different concentrations, if we start with sugar solution of concentration above $40 \%$, then relation between refractive index and concentration of sugar solution comes out to be non-linear at those high concentrations with respect to low concentration. But at low concentrations below $20 \%$ the relation is linear. This is because at high concentration sugar is dominating and at low concentration water is dominating. That means at high concentration about $80 \%$ the ref. index of the solution is near to that of sugar and at very low concentration the ref. index of solution is near to that of water. That means, at high concentrations about $80 \%$ and above you are measuring the refractive index of sugar rather than that of solution. Similarly at very low concentration $2.5 \%$ or near to it you are measuring refractive index of water. So, we make sugar solution of concentration equal and below $20 \%$ in our lab.

\section{Our trend for general physics practical filing}

In our traditional method of submission of practical reports, a general physics practical report consists:

1. Object of your experiment.

2. Apparatus required.

3. Theory.

4. Observations and calculations.

5. Error analysis.

6. Result of your experiment.

$7 . \quad$ Discussion.

\section{What should a standard physics lab report include?}

Actually, a standard physics lab report includes following requirements.

A. Experimental Description

1. Provide a statement of the physical theory or principle observed during the experiment.

2. Briefly describe the experiment you performed.

3. Discuss the relevance of the experiment to the theory.

B. Discussion of Graphs

1. For each graph, discuss the proximity of the data points to the line of best fit.

2. For each graph, state the theoretical values for slope and y-intercept.

3. For each graph, perform a percent difference 
calculation between the theoretical slope and $y$-intercept, and the measured values of slope and y-intercept.

C. Uncertainty Analysis Discussion

1. Discuss the method used to determine the measured uncertainty value for each measured quantity.

2. Consider each sub-experiment and determine the dominant source of uncertainty.

3. Determine the percent difference, percent uncertainty and percent variation. Then,

(a) Determine if each percent difference value is larger than or smaller than the dominant percent uncertainty for the experiment.

(b) Draw conclusions based on your findings; for example determine the factors contributing to percent difference values greater than the percent uncertainty value.

(c) Determine experimental factors which could have contributed to variation of measurements, i.e. how would you account for measured values that are not constant.

4. What did you think of the experiment and What would you do to improve the experiment?

D. Inquiry Based Lab Conclusions/ Discussion: Finally, predict the conclusions of your lab report. In particular, a paragraph should be written for each experiment. Each paragraph should contain the following information:

1. Prediction: What was the prediction that was made about the system?

2. Reasoning: What was the reasoning behind the prediction (i.e. why did you make the prediction that you made)?

3. Experimental Result: What was the result of the experiment?

4. Analysis:

(a) If the experiment agreed with you prediction, write a brief statement indicating this agreement.

(b) If the experiment did not agree with the prediction, discuss what was wrong with the reasoning that led to the prediction that you made.

\section{For Undergraduate Level}

In the contest of our domestic universities or boards, we are just adopting the method of percentage error

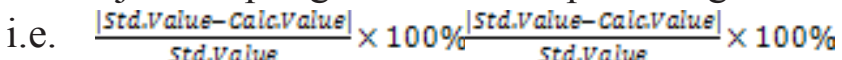
, even at bachelor level. This method is of very ground level and is suitable for +2 level students. So, bachelor level students at least should calculate the error on the result due to propagation of errors on different measurements as illustrated in below two examples and analysis/interpretation of the result should be done instead of writing just the points of precautions. This approach will make the students somewhat familiar about the error analysis on advanced level. Otherwise, the hesitation and difficulty about error analysis which stuns M.Sc. level beginner student will persist and the necessity of standardization of our lab reports will remain untouched for next several years also.

Ex.1: Determination of value of ' $\mathrm{g}$ ' by using simple pendulum.

Suppose: $l=75 \mathrm{cml}=75 \mathrm{~cm}$ by using meter scale. Then, $\Delta l=1 \mathrm{~mm} \Delta l=1 \mathrm{~mm}$. Suppose the time of 20 oscillations is 35 seconds measured with a wrist watch of $1 \mathrm{sec}$. resolution. Then, $g$ $=4 \pi^{2} \frac{l}{T^{2}}=9.668 \mathrm{~m} / \mathrm{s}^{2}=4 \pi^{2} \frac{1}{T^{2}}=9.668 \mathrm{~m} / \mathrm{s}^{2}$ and taking log, we have,

$\log g=\log 4 \pi^{2}+4 \pi^{2}+\log l-l-2 \log T$

Differentiating we get; $\frac{\Delta \mathrm{g}}{\mathrm{g}}=\frac{\Delta l}{l}-2 \frac{\Delta \mathrm{T}}{\mathrm{T}} \frac{\Delta \mathrm{g}}{\mathrm{g}}=\frac{\Delta l}{\mathrm{l}}-2 \frac{\Delta \mathrm{T}}{\mathrm{T}}$ Permissible error in $g g=\Delta g \Delta g=g\left(\frac{\Delta l}{l}+2 \frac{\Delta \mathrm{TS} l}{\mathrm{~T} l}+2 \frac{\Delta \mathrm{T}}{\mathrm{T}}\right)$ $=9.668\left(\frac{0.001}{0.75}+2 \frac{1}{35}\right) 9.668\left(\frac{0.001}{0.75}+2 \frac{1}{35}\right)=0.560 \mathrm{~m} / \mathrm{s}^{2} \mathrm{~m} / \mathrm{s}^{2}$

[Since, error is additive]

Relative error in $\mathrm{gg}=\frac{\Delta \mathrm{g}}{\mathrm{g}}=\frac{\Delta \mathrm{g}}{\mathrm{g}}=\frac{\Delta \mathrm{I}}{\mathrm{l}}+2 \frac{\Delta \mathrm{ISI}}{\mathrm{T} l}+2 \frac{\Delta \mathrm{I}}{\mathrm{T}}$

$=\left(\frac{0.001}{0.75}+2 \frac{1}{35}\right)=0.058$

$\therefore \%$ error on $g=\frac{\Delta g}{g} \times 100 \%=\left(\frac{\Delta l}{l}+2 \frac{\Delta T}{T}\right) \times 100 \%$ $=\left(\frac{0.001}{0.75}+2 \frac{1}{35}\right) \times 100 \%=5.87 \%$

Result: value of $g=\left(9.668 \pm(9.668 \pm 0.560) \mathrm{m} / \mathrm{s}^{2}\right.$ $\mathrm{m} / \mathrm{s}^{2}$ 
Ex.2: To determine the surface tension of given liquid at room temperature.

Suppose the quantities density ' $\mathrm{d}$ ', acceleration due to gravity ' $g$ ' and angle of contact $\theta \theta$ are taken from the table of constants, while height of liquid raised inside capillary tube and diameter of the capillary tube are measured as $\mathrm{h}=3.00 \pm \pm 0.01 \mathrm{~cm}$ and $\mathrm{D}=0.250 \pm \pm 0.001 \mathrm{~cm}$. Then, $\mathrm{T}=\frac{\text { Dhdg }^{4} \mathrm{Cos} \theta}{\mathrm{T}}=\frac{\text { Dhdg }}{4 \cos \theta}$ gives observed value for $\mathrm{T}$ where $\mathrm{h}=3.00 \mathrm{~cm}$ and $\mathrm{D}=0.250 \mathrm{~cm}$.

Permissible error in $\underline{\mathrm{TT}}=\Delta \mathrm{T} \Delta \mathrm{T}=\mathrm{T}\left(\frac{\Delta \mathrm{D}}{\mathrm{D}}+\frac{\Delta \mathrm{h} \Delta \mathrm{D}}{\mathrm{D}}+\frac{\Delta \mathrm{h}}{\mathrm{h}}\right)$ $\{\because \mathrm{d}, \mathrm{g}$ and $\theta$ are constants $\} \mathrm{d}, \mathrm{g}$ and $\theta$ are constants $\}$

[Here, $\Delta \mathrm{D}=0.001 \mathrm{~cm}$ and $\Delta \mathrm{h}=0.01 \mathrm{~cm}$ ]

$\Delta \mathrm{D}=0.001 \mathrm{~cm}$ and $\Delta \mathrm{h}=0.01 \mathrm{~cm}]$

Relative error in $\mathrm{T}=\frac{\Delta \mathrm{TST}}{\mathrm{T} T}=\left(\frac{\Delta \mathrm{D}}{\mathrm{D}}+\frac{\Delta \mathrm{h} \Delta \mathrm{D}}{\mathrm{h}}+\frac{\Delta \mathrm{h}}{\mathrm{h}}\right)$

$\therefore \%$ error on $\mathrm{T}=\frac{\Delta \mathrm{T}}{\mathrm{T}} \times 100 \%=\left(\frac{\Delta \mathrm{D}}{\mathrm{D}}+\frac{\Delta \mathrm{h}}{\mathrm{h}}\right) \times 100 \%$ $\frac{\Delta \mathrm{T}}{\mathrm{T}} \times 100 \%=\left(\frac{\Delta \mathrm{D}}{\mathrm{D}}+\frac{\Delta \mathrm{h}}{\mathrm{h}}\right) \times 100 \%$

Result: value of $g=T \pm \Delta T=\frac{D h d g}{4 \cos \theta} \pm \frac{D h d g}{4 \cos \theta}\left(\frac{\Delta D}{D}+\frac{\Delta h}{h}\right.$ $\mathrm{T} \pm \Delta \mathrm{T}=\frac{\mathrm{Dhdg}}{4 \cos \theta} \pm \frac{\mathrm{Dhdg}}{4 \cos \theta}\left(\frac{\Delta \mathrm{D}}{\mathrm{D}}+\frac{\Delta \mathrm{h}}{\mathrm{h}}\right)$

\section{Remarks}

1. Precession means how small you can measure and Accuracy means how correct you can measure.

2. Taylor's series expansion to calculate $Z\left(A_{0}+\Delta A\right)=Z\left(A_{0}\right)+\Delta A\left(\frac{\delta Z}{\delta A}\right)_{A=A_{0}}+\cdots+\cdots$

Or, $\Delta z=z\left(A_{0}+\Delta A\right)-z\left(A_{0}\right) \Delta z=z\left(A_{0}+\Delta A\right)-z\left(A_{0}\right)$

Or, $\Delta Z \approx \Delta A\left(\frac{\delta Z}{\partial A}\right)_{A=A_{0}} \Delta Z \approx \Delta A\left(\frac{\delta Z}{\delta A}\right)_{A=A_{0}}$

$\rightarrow \Delta V=\Delta l\left(\frac{\delta V}{\delta l}\right)_{l=l_{0}}=\left[3 \Delta l \cdot l^{2}\right]_{l=l_{0}}$

Or, $\frac{\Delta V}{V}=3 \frac{\Delta L V V}{l_{0} V}=3 \frac{\Delta l}{l_{0}}$

3. Direct method of series expansion to calculate error:

Ex: $\underline{V(l)=l^{2} V(l)=l^{2}}$

$\therefore V(l+\Delta l)=(l+\Delta l)^{3}=l^{3}+3 l^{2} \Delta l+3 l(\Delta l)^{2}+(\Delta l)^{3}$

Or, $\quad \Delta V=V(l+\Delta l)-V(l)=3 l^{2} \Delta l+3 l(\Delta l)^{2}+(\Delta l)^{3}$

$\Delta V=V(l+\Delta l)-V(l)=3 l^{2} \Delta l+3 l(\Delta l)^{2}+(\Delta l)^{3}$

4. Logarithmic method to calculate $\%$ error (for small \% change of a factor only):
Ex: A bulb is rated at $100 \mathrm{~V}, 220 \mathrm{~W}$. When the voltage drops

$\frac{\Delta P}{P} \times 100 \%=\frac{\left|P^{\prime}-P\right|}{P} \times 100 \%=\frac{\left|\frac{V^{2}}{R}-\frac{V^{2}}{R}\right|}{\frac{V^{2}}{R}} \times 100 \%=\frac{\left|\frac{9 g^{2}}{R}-\frac{100^{2}}{R}\right|}{\frac{100^{2}}{R}} \times 100 \%=4 \%$

But, by taking logarithm on both sides of: $P=\frac{V^{2}}{R}$ $\frac{\bar{\Delta} P}{P}=2 \frac{V^{2}}{V} ;$ since $R$ is constant

Or, $\quad \frac{\Delta P}{P} \times 100 \%=2 \frac{\Delta V}{V} \times 100 \%=2 \times 2 \%=4 \%$ $\frac{\Delta P}{P} \times 100 \%=2 \frac{\Delta V}{V} \times 100 \%=2 \times 2 \%=4 \%$

\section{References}

Numerical Mathematical Analysis, James B. Scarborough; Oxford \& IBH Publishing Co. Pvt. Ltd.

Taylor, John. An Introduction to Error Analysis, 2nd. ed. University Science Books: Sausalito. Baird, D.C. Experimentation: An Introduction to Measurement Theory and Experiment Design, Prentice Hall: Englewood Cliffs.

Bevington, Phillip and Robinson, D. Data Reduction and Error Analysis for the Physical Sciences, McGraw-Hill: New York.

ISO. Guide to the Expression of Uncertainty in Measurement. International Organization for Standardization (ISO) and the International Committee on Weights and Measures (CIPM): Switzerland, 1993.

Physics Lab Report Guidelines; A special publication of Mercer University.

Workshop on Physics Laboratory Course of M.Sc. Level at T.U.' held on Dept. of Physics, Amrit Campus, Kathmandu, on 28-30 of January2011. 